\title{
Effect of preoperative anxiety, depression, and insomnia on acute postoperative pain after non- cardiac surgery
}

Dandan Lin

Capital Medical University Affiliated Beijing Chaoyang Hospital

\section{Xiao Huang}

Beijing Chaoyang Hospital

Jing Wang

Beijing Chaoyang Hospital

Yanan Hao

Beijing Chaoyang Hospital

Mengwen Geng

Beijing Chaoyang Hospital

Changwei Wei ( $\square$ changwei.wei@ccmu.edu.cn )

Beijing Chaoyang Hospital https://orcid.org/0000-0001-5652-600X

\section{Research article}

Keywords: anxiety, depression, insomnia, postoperative pain, non-cardiac surgery

Posted Date: April 20th, 2021

DOI: https://doi.org/10.21203/rs.3.rs-429163/v1

License: (9) (i) This work is licensed under a Creative Commons Attribution 4.0 International License.

Read Full License 


\section{Abstract \\ Background}

The mental diseases especially anxiety, depression, and insomnia are common in patients at perioperative period. This study aims to investigate the association of preoperative mental diseases with acute postoperative pain of patients undergoing non-cardiac surgery.

\section{Method:}

The patients undergoing non-cardiac surgery from August 1, 2019 to January 23, 2020 at Capital Medical University, Beijing Chao-Yang Hospital were included in this retrospective study. The demographics, clinical data and assessments of depression, anxiety, and insomnia by Insomnia Severity Index (ISI), Patient Health Questionnaire-9 (PHQ-9), Generalized Anxiety Disorder-7 (GAD-7) were collected and analyzed. The patients with any of depression, anxiety or insomnia were divided into mental disease group, and others were in non-mental disease group according to the. We compared the postoperative visual analog score between patients in mental diseases group with propensity score matching cohort from patients in non-mental diseases. The primary outcome was acute postoperative pain according to the Visual Analog Scale (VAS) 24h after surgery. Secondary outcomes included VAS at other time points and incidence of postoperative nausea and vomiting (PONV), rescue analgesia, and length of hospital stay (LOS).

\section{Results}

The analysis included 397 patients (274 in the group and 123 in the mental disease group). Patients in mental disease group (anxiety, depression and insomnia) were associated with higher VAS scores and increased times of rescue analgesia. Each of the 3 mental diseases was associated with higher VAS scores independently.

\section{Conclusion}

In this retrospective study, preoperative anxiety, depression and insomnia were associated with increased the level of acute postoperative pain and increased times of rescue analgesia of patients undergoing noncardiac surgery.

\section{Plain English Summary}

The mental diseases including anxiety, depression and insomnia are common in patients at perioperative period. And the association between the mental disease and postoperative acute pain remains unclear. We conduct this cohort study to assess the association of mental disease and postoperative acute pain 
in patients undergoing non-cardiac surgery. We hypothesize the patients with mental disease will tolerate severe postoperative pain at $24 \mathrm{~h}$ after surgery. The results are that patients in mental disease group (anxiety, depression and insomnia) were associated with higher VAS scores at $24 \mathrm{~h}, 48 \mathrm{~h}$ and $72 \mathrm{~h}$ after surgery, and increased times of rescue analgesia. Each of the 3 mental diseases was associated with higher VAS scores independently. These indicate that patients with mental diseases suffered more severe postoperative pain than patients without mental disease. The funding of this study inspire that physicians should pay more attention and give enough rescue analgesia to these patients.

\section{Background}

Mental disease is a disorder leading to different degrees of impairment in cognitive behavior and other mental activities as clinical manifestations[1, 2]. More than 1 billion people worldwide were affected by the mental disease in 2016, and they contributed to $7 \%$ of the global burden of all diseases[3]. About $14.3 \%$ of deaths worldwide, or about 8 million people per year, can be attributed to mental disorders[4]. In recent years, the prevalence of mental diseases in perioperative patients increased over time [5]. The most common mental disease in the perioperative period are anxiety, depression and insomnia and the prevalence of them are $21.9 \%, 14.1 \%$, and $17.8 \%[6,7]$. Mental disease in surgical patients could lead to negative short-term and long-term outcomes after surgery and a significant decrease in quality of life [8, 6]. Surgical patients with severe mental disease present a significant challenge to clinicians.

Pain harms the quality of life $[9,10]$, and adequate analgesia is essential to improve postoperative recovery $[11,12]$. Risk factors for postoperative pain include genetic susceptibility, preceding pain, psychosocial factors, age and gender[13]. Psychological factors play an important role in non-surgical causes of pain[14]. Memories of pain, fear and related emotions such as anxiety, depression and insomnia can influence the response to noxious stimuli. And pain may be the result of the interaction of biological and psychological variables. Kain et al. showed preoperative anxiety in young children increased incidence of postoperative pain and sleep problems[15]. However, another study conducted by Wong et al. found that the presence of preoperative psychiatric disease did not worsen postoperative outcomes[16]. The results of a meta-analysis showed that preoperative pain, mental disease, anxiety, depression, and somatoform disorders had a detrimental effect on postoperative pain and function after total knee arthroplasty (TKA) [17]. Although some relevant studies exist, there is controversy regarding the impact of preoperative mental disease on pain. And the effect of preoperative mental disease on acute postoperative pain in non-cardiac patients remains unclear. Due to the adverse effects of mental illness on surgical outcomes, we hypothesize that patients with preoperative mental disease will tolerate more severe acute postoperative pain. Therefore, we conducted this cohort study to assess the association of mental disease and postoperative acute pain in surgical patients.

\section{Methods}

\section{Patient selection and data extraction}


The patients were enrolled between September 2019 and January 2020. The study was approved by the Regional Ethical Review Committee at the Beijing Chao-Yang Hospital, Capital Medical University approved this retrospective study (2019-ke-273).

Patients were included according to the following inclusion criteria: (1) 18 years or older;(2) American

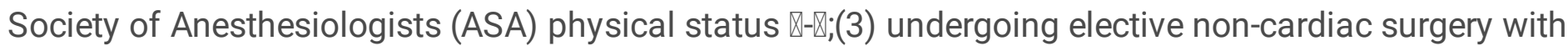
general anesthesia at Beijing Chao-Yang Hospital. Exclusion criteria were as follows: (1) history of dementia or severe cognitive impairment;(2) history of the psychiatric disease;(3) history of organic sleep disorder ;(4) taking antipsychotic medication; (4) history of drug abuse.

\section{Data collection}

We collected the patient's general information and preoperative clinical data including gender, age, body mass index (BMI), smoking history, alcohol consumption, years of education, preoperative comorbidities (coronary artery disease, hypertension, diabetes), preoperative Visual Analog Scale (VAS) score. We also reviewed and analyzed patients' assessment of depression, anxiety, and insomnia using the Insomnia Severity Index (ISI), Patient Health Questionnaire-9 (PHQ-9), Generalized Anxiety Disorder-7 (GAD-7) respectively one day before surgery.

The surgery and anesthesia data including ASA physical status classification, type of surgery, duration of surgery, anesthesia types, and postoperative analgesia were also collected. We included postoperative data including VAS scores in the postoperative anesthesia recovery room (PACU), 24, 36, 48, 60, 72, and 84 hours after surgery respectively. Besides, we recorded postoperative nausea and vomiting (PONV), rescue analgesia, and length of hospital stay (LOS). The assessments of ISI, PHQ-9 and GAD-7 were extracted from the paper-based questionnaire. Other data were extracted from electronic medical records.

\section{Exposure of interest}

For this study, we identified the mental disease according to the scores of ISI, PHQ-9 and GAD-7. The ISI used a cut-off value of 8 points, with a score $\geq 8$ points being insomnia and less than 8 points being normal[18]. And 5 points was used as the cut-off value for both the PHQ-9 and GAD-7, with a higher score indicating moderate to severe depression or anxiety $[19,20]$. Patients were separated into non-mental diseases group and mental diseases group (with anxiety, depression or insomnia) based on the assessments.

\section{Outcomes}

We compared the effect of preoperative mental disease on postoperative pain. And propensity score matching (PSM) analysis was performed to reduce selection bias. The primary outcome was postoperative pain according to VAS $24 \mathrm{~h}$ after surgery. Secondary outcomes included VAS at other time points and incidence of PONV, rescue analgesia, and LOS.

\section{Statistical analysis}


Statistical analysis was performed with IBM SPSS Statistics version 25.0 (IBM Corp., Armonk, NY, USA). Continuous variables are expressed as a median and interquartile range for non-normal distribution variables and mean values with standard deviation for normal distribution variable. Categorical variables were expressed as counts and percentages. We compared the categorical variables with the chi-square test or Fisher's exact test. Levene's test was used to test for equality of variances of continuous variables. Independent t-tests were performed for continuous variables with symmetric distributions. And the Mann Whitney $\mathrm{U}$ test was used to compare continuous data with the asymmetric distribution.

We performed PSM analysis using multivariable logistic regression model by R (R Foundation for Statistical Computing, version 3.5.1) to build matched groups of patients to compare postoperative acute pain between patients with or without any of mental diseases. Variables were identified in the present study. These variables used for matching included ASA physician status, surgery site, preoperative pain. The matched group of patients from non-mental disease group were in Group $A$ and the matched patients from mental disease group were in Group B. To investigate the effect of each mental diseases (anxiety, depression and insomnia), PSM were used which variables were also included ASA physician status, surgery site, preoperative pain. When we analyzed 1 mental disease, the other 2 mental diseases were also included in variables in order to minimize their effects. Matching analysis was performed based on each patient's estimated propensity score using a nearest neighbor 1:1 matching with a caliper range of 0.2. We tested the patients demographic and perioperative characteristics of patients before and after matching to ensure well-matching. Two-sided $P$ values of less than 0.05 were regarded to be of statistical significance.

\section{Results}

From August 1, 2019 to January 23, 2020, a total of 400 patients' electronic medical records were screened. Among these patients, 3 patients were excluded because of their incompletely medical records. Then, 397 patients undergoing non-cardiac surgery were enrolled and analyzed. The flow diagram was shown in Fig. 1.

The demographic and perioperative characteristics of the 397 enrolled patients were listed in Table 1. There were 170 males and 227 females enrolled. Of these, 40 underwent head and neck surgery, 80 underwent thoracic surgery, 211 underwent abdominal surgery and 67 underwent joint replacement and spinal surgery. And all of the patients were used general anesthesia. 
Table 1

Demographic characteristics and perioperative data of the patients enrolled

\begin{tabular}{|c|c|c|c|c|}
\hline & Total & $\begin{array}{l}\text { Non-mental } \\
\text { disease }\end{array}$ & $\begin{array}{l}\text { Mental } \\
\text { disease }\end{array}$ & \\
\hline Characteristic & $(n=397)$ & $(n=274)$ & $(n=123)$ & $P$ \\
\hline \multicolumn{5}{|l|}{ Demographic characteristics } \\
\hline Male, n (\%) & $\begin{array}{l}170 \\
(42.1)\end{array}$ & $125(45.6)$ & $45(36.5)$ & 0.080 \\
\hline Age (y) & $\begin{array}{l}55.2 \pm \\
14.0\end{array}$ & $54.6 \pm 14.1$ & $56.4 \pm 13.7$ & 0.233 \\
\hline $\mathrm{BMI}\left(\mathrm{kg} / \mathrm{m}^{2}\right)$ & $24.7 \pm 3.7$ & $24.9 \pm 3.7$ & $24.2 \pm 3.7$ & 0.094 \\
\hline History of smoking, n (\%) & $\begin{array}{l}110 \\
(27.7)\end{array}$ & $77(28.1)$ & $31(25.2)$ & 0.816 \\
\hline Alcohol consumption, n (\%) & $81(20.4)$ & $58(21.1)$ & $23(18.7)$ & 0.815 \\
\hline Education year, (y) & $11.2 \pm 3.7$ & $10.6 \pm 3.7$ & $11.5 \pm 3.6$ & 0.083 \\
\hline \multicolumn{5}{|l|}{ Medical history } \\
\hline CAD, n (\%) & $7(1.7)$ & $4(1.4)$ & $3(2.4)$ & 0.422 \\
\hline Hypertension, n (\%) & $\begin{array}{l}137 \\
(34.5)\end{array}$ & $92(33.7)$ & $45(36.5)$ & 0.611 \\
\hline DM, n (\%) & $64(16.1)$ & $38(13.9)$ & $26(21.1)$ & 0.079 \\
\hline Preoperative VAS & $0(0,0)$ & $0(0,0)$ & $0(0,1)$ & 0.005 \\
\hline ASA & & & & 0.031 \\
\hline ० & $53(13.3)$ & $45(16.4)$ & $8(6.5)$ & \\
\hline ૧ & $255(64.2)$ & $173(63.4)$ & $82(66.7)$ & \\
\hline$\square$ & $89(22.5)$ & $56(20.4)$ & $33(26.8)$ & \\
\hline \multicolumn{5}{|l|}{ Surgical characteristics } \\
\hline Surgical site, n (\%) & & & & 0.009 \\
\hline Head and neck & $40(10.1)$ & 26(9.5) & 14(11.4) & \\
\hline Chest & $80(20.2)$ & 47(17.2) & $33(26.8)$ & \\
\hline Abdomen & 211(53.1) & $160(58.6)$ & $51(41.5)$ & \\
\hline
\end{tabular}

Legend of Table 1. BMI, body mass index; CAD, coronary artery disease; DM, diabetes mellitus; ASA, American Society of Anesthesiologists; TIVA, total intravenous anesthesia; PCIA, patient-controlled intravenous analgesia; PCEA, patient-controlled epidural analgesia. 


\begin{tabular}{|c|c|c|c|c|}
\hline & Total & $\begin{array}{l}\text { Non-mental } \\
\text { disease }\end{array}$ & \multicolumn{2}{|l|}{$\begin{array}{l}\text { Mental } \\
\text { disease }\end{array}$} \\
\hline Arms and legs & 67(13.4) & $41(15.0)$ & \multicolumn{2}{|l|}{$25(20.3)$} \\
\hline Surgery duration (min) & $\begin{array}{l}139.8 \pm \\
85.5\end{array}$ & $135.3 \pm 83.0$ & $149.8 \pm 90.3$ & 0.211 \\
\hline \multicolumn{4}{|l|}{ Anesthesia } & 0.308 \\
\hline TIVA & $254(64.0)$ & $178(65.0)$ & \multicolumn{2}{|l|}{$76(61.8)$} \\
\hline $\begin{array}{l}\text { Intravenous and inhalational balanced } \\
\text { anesthesia }\end{array}$ & $110(27.8)$ & $76(27.7)$ & \multicolumn{2}{|l|}{$35(28.5)$} \\
\hline Intravenous and nerve block & 15(3.9) & $8(2.9)$ & \multicolumn{2}{|l|}{$7(5.7)$} \\
\hline Intravenous and intraspinal anesthesia & $18(4.3)$ & $12(4.4)$ & \multicolumn{2}{|l|}{$5(4.0)$} \\
\hline \multicolumn{4}{|l|}{ Analgesia } & \multirow[t]{2}{*}{0.575} \\
\hline PCIA & 198(49.9) & $131(47.8)$ & $67(54.5)$ & \\
\hline PCEA & $29(7.3)$ & $22(8.0)$ & \multicolumn{2}{|l|}{$7(5.7)$} \\
\hline None & $172(42.8)$ & $121(44.2)$ & \multicolumn{2}{|l|}{$49(39.8)$} \\
\hline $\begin{array}{l}\text { Legend of Table 1. BMI, body mass inde } \\
\text { American Society of Anesthesiologists; } \\
\text { intravenous analgesia; PCEA, patient-co }\end{array}$ & $\begin{array}{l}\text { D, coronary } \\
\text { total intrave } \\
\text { ed epidural }\end{array}$ & $\begin{array}{l}\text { tery disease; } \\
\text { ous anesthesi } \\
\text { algesia. }\end{array}$ & $\begin{array}{l}\text { abetes mellitus } \\
\text { A, patient-contr }\end{array}$ & $\begin{array}{l}\text { ASA, } \\
\text { led }\end{array}$ \\
\hline
\end{tabular}

The incidence of anxiety, depression and insomnia was $13.4 \%, 13.6 \%$ and $15.1 \%$ respectively. Patients were divided into two groups according to the preoperative assessments of mental disease (274 in nonmental disease group with mental disease and 123 in mental disease group without any mental disease). The demographic and perioperative characteristics were shown in Table 1. No significant differences were found between the two groups in basic characteristics, including gender, age, BMI, history of smoking, alcohol consumption, education years, comorbidities, preoperative VAS score, surgery duration,

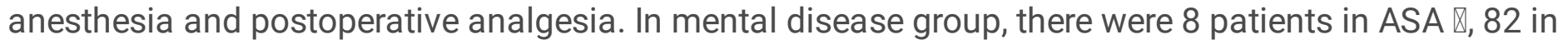

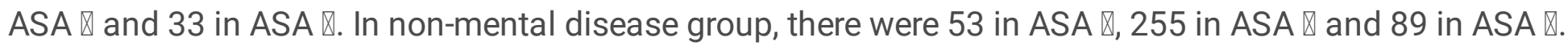

And postoperative outcomes after PSM were shown in Table 2. Compared with group A, the VAS score was higher in PACU, 24, 48 and 72 hours after surgery in group $B(P<0.05)$. There were no significant differences in the incidence of PONV and LOS. The times of rescue analgesia was higher in group $B$ than that of group $\mathrm{A}(P<0.05)$. 
Table 2

Postoperative outcomes of 2 groups

\begin{tabular}{|c|c|c|}
\hline & $\begin{array}{l}\text { Group A } \\
(n=119)\end{array}$ & $\begin{array}{l}\text { Group B } \\
(n=123)\end{array}$ \\
\hline \multicolumn{3}{|l|}{ Postoperative VAS } \\
\hline PACU & $2(0,3)$ & $2(1,3)^{*}$ \\
\hline $24 \mathrm{~h}$ & $2(1,3)$ & $3(1,4)^{*}$ \\
\hline $36 \mathrm{~h}$ & $2(1,3)$ & $2(0,4)$ \\
\hline $48 \mathrm{~h}$ & $2(0,3)$ & $2(0,3)^{*}$ \\
\hline $60 \mathrm{~h}$ & $1(0,3)$ & $2(0,3)$ \\
\hline $72 \mathrm{~h}$ & $1(0,2)$ & $2(0,3)^{*}$ \\
\hline $84 \mathrm{~h}$ & $1(0,2)$ & $1(0,3)$ \\
\hline PONV & 51 & 25 \\
\hline Rescue analgesia & 64 & 42 \\
\hline LOS & $9(6,13)$ & $8(6,12)$ \\
\hline
\end{tabular}

According to the propensity scored matched, the patients were divided into 3 pair groups, anxiety and non-anxiety group, depression and non-depression group, insomnia and non-insomnia group. The postoperative VAS scores were shown in Fig. 2. Compared with the non-anxiety group, the VAS score was higher in $\operatorname{PACU}(P<0.05)$. Compared with the non-depression group, the VAS score was higher in PACU and 24 hours after surgery $(P<0.05)$. Compared with the non-insomnia group, the VAS score was higher in PACU, 48 and 72 hours after surgery in the insomnia group $(P<0.05)$.

\section{Discussion}

In this study, we found that mental diseases were negative associated with acute postoperative pain on patients undergoing non-cardiac surgery after matching the ASA physical status, surgery site and preoperative pain level. Furthermore, anxiety, depression and insomnia could increase the pain postoperative pain level at different time points. The association was held after propensity score matching. Besides, the mental diseases would increase the times of rescue analgesia.

Consistent with the previous study, psychological diseases are associated with higher pain levels[21]. Various psychological mechanisms may play an important role in central sensitization[22]. An 
association between anxiety and depression and postoperative pain was described by Gravani, where an increased intensity and higher level of pain at 1 and 4 hours after surgery were found for patients with anxiety and depression undergoing bariatric surgery[23]. But contrasted with our study, no significance was found in pain level 24 hours after surgery. In this study, we found the VAS scores were still higher in 24, 48 and 72 hours after surgery in patients with anxiety, depression and insomnia when compared with non-mental diseases patients. And the effect of mental diseases on acute postoperative pain could last longer after surgery. Kulkarini reported anxiety and depression were associated with postoperative pain 1 week after surgery[24].

Furthermore, in our study, we were first to included insomnia as one of the essential perioperative mental diseases with anxiety and depression and to analyze the association with acute postoperative pain. Previously, insomnia was thought to be a symptom of anxiety and depression. However, insomnia plays an important role in surgical patients' short-term and long-term outcomes[25, 26]. Consistent with OrbachZinger's study, insomnia increased the severe pain upon movement[27]. A limitation of the study is that the included patients were all female undergoing cesarean surgery, which might be affected by multiple factors including family factors, disturbance of taking care of babies. We also found the negative effect of insomnia on postoperative pain after carefully propensity matching of ASA, preoperative pain, surgery site, anxiety, and depression. And in Luo's study, they reported, the effect of insomnia on postoperative pain could last to 12 weeks after surgery for patients undergoing total joint arthroplasty [28].

Besides, in group A, the pain level decreased significantly after 24 hours after surgery. But this trend was slowly and moved to longer than 72 hours after surgery. We were the first to report the VAS scores at consistent time points after surgery. The results will help clinicians make better analgesia management of patients who will benefit from sufficient analgesia and increase hospitalization satisfaction.

Previously, there was evidence of increased risk of postoperative nausea in surgical patients with depression symptoms[29]. In contrast with the previous study, we didn't find differences in the incidence of PONV between the two groups. NSAIDs drug in rescue analgesia treatment may be more pronounced among high-need rescue analgesia patients undergoing non-cardiac surgery. In our study, the times of rescue analgesia in the mental diseases group were more than that of group A after PSM. This was also indicated that the patients suffered more pain after surgery.

Besides, we found that the length of LOS in the mental diseases group was shorter than that of group A. The patients who we included were found the psychiatric change in the perioperative period. Lots of hospital-related risk factors contributed to this change and patients might found it difficult to adapt hospital environment[5]. After the surgery, patients would be more likely to discharge early.

Our study has several limitations. First, this study was a single-center retrospective observational study. Second, although the propensity scores balanced ASA, surgery site, preoperative pain and mental diseases, there might be other unbalanced cofounding factors about the mental disease. These included the duration and severity of the mental disease. In this study, we simply assessed patients for preoperative anxiety, depression, or insomnia as having a mental disease. We understand that mental 
disease can present with other psychological states that are not limited to the above three. However, we believe that anxiety, depression, and insomnia are common in surgical patients, and studying their effects with acute postoperative pain may also serve as a basis for future research. And further studies are needed to explore the correlation between the different severity, types, and duration of mental disease and acute postoperative pain.

\section{Conclusion}

In summary, we found an association between the preoperative mental diseases with the increased level of acute postoperative pain of patients undergoing non-cardiac surgery. Further work should focus on the potential mechanisms and how to identify the patients at high risk of mental diseases. And more investigations are required the physicians should take more attention to the perioperative pain management of patients with mental diseases to improve their postoperative recovery quality and satisfaction of hospitalization.

\section{Abbreviations}

ASA: American Society of Anesthesiologists; BMI: Body Mass Index; GAD-7: Generalized Anxiety Disorder7; ISI: Insomnia Severity Index; LOS: Length of Hospital Stay; PACU: Postoperative Anesthesia Recovery Room; PHQ-9: Patient Health Questionnaire-9; PONV: Postoperative Nausea and Vomiting; PSM: propensity score matching; TKA: Total Knee Arthroplasty; VAS, Visual Analog Scale.

\section{Declarations}

\section{Ethics approval and consent to participate}

The study was approved by the Regional Ethical Review Committee at the Beijing Chao-Yang Hospital, Capital Medical University approved this retrospective study (2019-ke-273). The written informed consents were obtained from participants if the participants meet the inclusion criteria.

\section{Consent to publish}

Not applicable.

\section{Availability of data and material}

The data could be acquired from corresponding author by reasonable requirements.

\section{Competing interest}

The authors declare that they have no conflict of interest.

\section{Funding}


This word was supported by Beijing Hospitals Authority Youth Programmed (QML 20190307). The funder had no role in the study implementation, data collection, data analyses or interpretation of study results.

\section{Authors' contribution:}

Study design: all authors; Collect data: JW, MG; Analysis of data: XH, DL; Result interpretation: JW, YH, $\mathrm{DL}, \mathrm{XH}$; Drafting of the manuscript: $\mathrm{DL}, \mathrm{XH}$; Critical revision of the manuscript: ASW, WCW.

\section{Authors' information}

1 Department of Anesthesiology, Beijing Chao-Yang Hospital, Capital Medical University, Beijing, China.

\section{Acknowledgements}

Not applicable.

\section{References}

1. Vigo, D., Thornicroft, G., \& Atun, R. (2016). Estimating the true global burden of mental illness. Lancet Psychiatry, 3(2), 171-178, doi:10.1016/s2215-0366(15)00505-2.

2. Steel, Z., Marnane, C., Iranpour, C., Chey, T., Jackson, J. W., Patel, V., et al. (2014). The global prevalence of common mental disorders: a systematic review and meta-analysis 1980-2013. Int J Epidemiol, 43(2), 476-493, doi:10.1093/ije/dyu038.

3. Rehm, J., \& Shield, K. D. (2019). Global Burden of Disease and the Impact of Mental and Addictive Disorders. Curr Psychiatry Rep, 21(2), 10, doi:10.1007/s11920-019-0997-0.

4. Walker, E. R., McGee, R. E., \& Druss, B. G. (2015). Mortality in mental disorders and global disease burden implications: a systematic review and meta-analysis. JAMA Psychiatry, 72(4), 334-341, doi:10.1001/jamapsychiatry.2014.2502.

5. Pan, X., Wang, J., Lin, Z., Dai, W., \& Shi, Z. (2019). Depression and Anxiety Are Risk Factors for Postoperative Pain-Related Symptoms and Complications in Patients Undergoing Primary Total Knee Arthroplasty in the United States. J Arthroplasty, 34(10), 2337-2346, doi:10.1016/j.arth.2019.05.035.

6. Takagi, H., Ando, T., \& Umemoto, T. (2017). Perioperative depression or anxiety and postoperative mortality in cardiac surgery: a systematic review and meta-analysis. Heart Vessels, 32(12), 14581468, doi:10.1007/s00380-017-1022-3.

7. Serbest, S., Tiftikçi, U., Askın, A., Yaman, F., \& Alpua, M. (2017). Preoperative and post-operative sleep quality evaluation in rotator cuff tear patients. Knee Surg Sports Traumatol Arthrosc, 25(7), 21092113, doi:10.1007/s00167-016-4228-5.

8. McBride, K. E., Solomon, M. J., Steffens, D., Bannon, P. G., \& Glozier, N. (2019). Mental illness and surgery: do we care? ANZ J Surg, 89(6), 630-631, doi:10.1111/ans.15248.

9. Billot, M., Daycard, M., Wood, C., \& Tchalla, A. (2019). Reiki therapy for pain, anxiety and quality of life. BMJ Support Palliat Care, 9(4), 434-438, doi:10.1136/bmjspcare-2019-001775. 
10. Neufeld, N. J., Elnahal, S. M., \& Alvarez, R. H. (2017). Cancer pain: a review of epidemiology, clinical quality and value impact. Future Oncol, 13(9), 833-841, doi:10.2217/fon-2016-0423.

11. Tang, W. K., Lau, C. G., Mok, V., Ungvari, G. S., \& Wong, K. S. (2015). The impact of pain on healthrelated quality of life 3 months after stroke. Top Stroke Rehabil, 22(3), 194-200, doi:10.1179/1074935714z.0000000024.

12. Govrin-Yehudain, O., Matanis, Y., \& Govrin-Yehudain, J. (2018). Reduced Pain and Accelerated Recovery Following Primary Breast Augmentation With Lightweight Breast Implants. Aesthet Surg J, 38(10), 1092-1096, doi:10.1093/asj/sjy071.

13. Kehlet, H., Jensen, T. S., \& Woolf, C. J. (2006). Persistent postsurgical pain: risk factors and prevention. Lancet, 367(9522), 1618-1625, doi:10.1016/s0140-6736(06)68700-x.

14. Mills, S. E. E., Nicolson, K. P., \& Smith, B. H. (2019). Chronic pain: a review of its epidemiology and associated factors in population-based studies. Br J Anaesth, 123(2), e273-e283, doi:10.1016/j.bja.2019.03.023.

15. Kain, Z. N., Mayes, L. C., Caldwell-Andrews, A. A., Karas, D. E., \& McClain, B. C. (2006). Preoperative anxiety, postoperative pain, and behavioral recovery in young children undergoing surgery. Pediatrics, 118(2), 651-658, doi:10.1542/peds.2005-2920.

16. Wong, S. E., Colley, A. K., Pitcher, A. A., Zhang, A. L., Ma, C. B., \& Feeley, B. T. (2018). Mental health, preoperative disability, and postoperative outcomes in patients undergoing shoulder arthroplasty. $J$ Shoulder Elbow Surg, 27(9), 1580-1587, doi:10.1016/j.jse.2018.02.066.

17. Sorel, J. C., Veltman, E. S., Honig, A., \& Poolman, R. W. (2019). The influence of preoperative psychological distress on pain and function after total knee arthroplasty: a systematic review and meta-analysis. Bone Joint J, 101-b(1), 7-14, doi:10.1302/0301-620x.101b1.bjj-2018-0672.r1.

18. Bastien, C. H., Vallieres, A., \& Morin, C. M. (2001). Validation of the Insomnia Severity Index as an outcome measure for insomnia research. Sleep Med, 2(4), 297-307, doi:10.1016/s13899457(00)00065-4.

19. Kroenke, K., Spitzer, R. L., \& Williams, J. B. (2001). The PHQ-9: validity of a brief depression severity measure. J Gen Intern Med, 16(9), 606-613, doi:10.1046/j.1525-1497.2001.016009606.x.

20. Spitzer, R. L., Kroenke, K., Williams, J. B., \& Lowe, B. (2006). A brief measure for assessing generalized anxiety disorder: the GAD-7. Arch Intern Med, 166(10), 1092-1097, doi:10.1001/archinte.166.10.1092.

21. Carr, E. C., Nicky Thomas, V., \& Wilson-Barnet, J. (2005). Patient experiences of anxiety, depression and acute pain after surgery: a longitudinal perspective. Int J Nurs Stud, 42(5), 521-530, doi:10.1016/j.ijnurstu.2004.09.014.

22. Skou, S. T., Graven-Nielsen, T., Rasmussen, S., Simonsen, O. H., Laursen, M. B., \& Arendt-Nielsen, L. (2014). Facilitation of pain sensitization in knee osteoarthritis and persistent post-operative pain: a cross-sectional study. Eur J Pain, 18(7), 1024-1031, doi:10.1002/j.1532-2149.2013.00447.x.

23. Gravani, S., Matiatou, M., Nikolaidis, P. T., Menenakos, E., Zografos, C. G., Zografos, G., et al. (2020). Anxiety and Depression Affect Early Postoperative Pain Dimensions after Bariatric Surgery. J Clin 
Med, 10(1), doi:10.3390/jcm10010053.

24. Kulkarni, A. R., Pusic, A. L., Hamill, J. B., Kim, H. M., Qi, J., Wilkins, E. G., et al. (2017). Factors Associated with Acute Postoperative Pain Following Breast Reconstruction. JPRAS Open, 11, 1-13, doi:10.1016/j.jpra.2016.08.005.

25. Wang, H., Zhang, L., Zhang, Z., Li, Y., Luo, Q., Yuan, S., et al. (2020). Perioperative Sleep Disturbances and Postoperative Delirium in Adult Patients: A Systematic Review and Meta-Analysis of Clinical Trials. Front Psychiatry, 11, 570362, doi:10.3389/fpsyt.2020.570362.

26. Su, X., \& Wang, D. X. (2018). Improve postoperative sleep: what can we do? Curr Opin Anaesthesiol, 31(1), 83-88, doi:10.1097/aco.0000000000000538.

27. Orbach-Zinger, S., Fireman, S., Ben-Haroush, A., Karoush, T., Klein, Z., Mazarib, N., et al. (2017). Preoperative sleep quality predicts postoperative pain after planned caesarean delivery. Eur J Pain, 21(5), 787-794, doi:10.1002/ejp.980.

28. Luo, Z. Y., Li, L. L., Wang, D., Wang, H. Y., Pei, F. X., \& Zhou, Z. K. (2019). Preoperative sleep quality affects postoperative pain and function after total joint arthroplasty: a prospective cohort study. $J$ Orthop Surg Res, 14(1), 378, doi:10.1186/s13018-019-1446-9.

29. Rodrigues, H. F., Furuya, R. K., Dantas, R. A. S., Rodrigues, A. J., \& Dessotte, C. A. M. (2018). Association of preoperative anxiety and depression symptoms with postoperative complications of cardiac surgeries. Rev Lat Am Enfermagem, 26, e3107, doi:10.1590/1518-8345.2784.3107.

\section{Figures}




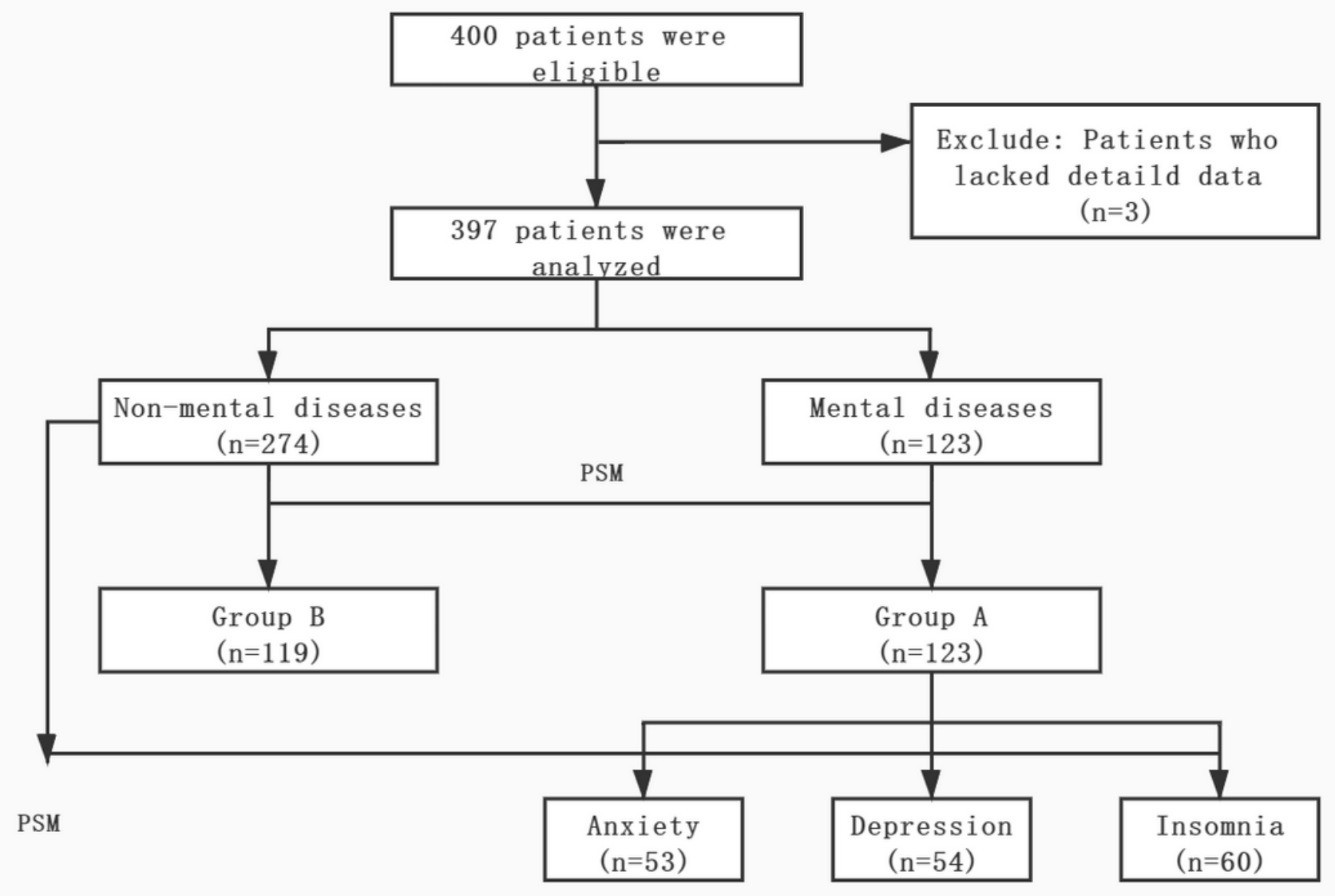

Figure 1

Flow diagram of the study. PSM: propensity score matching. 
A.

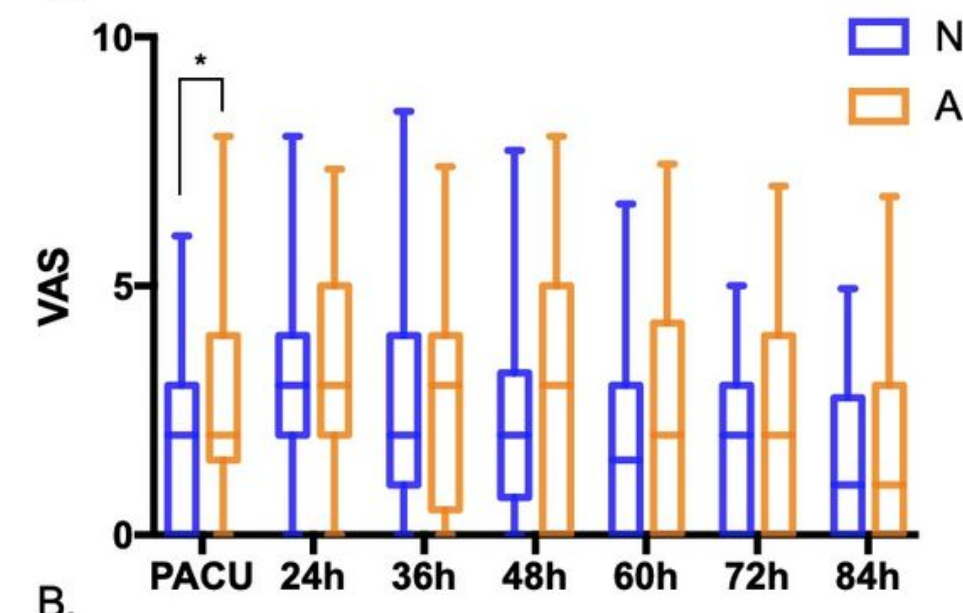

Non-anxiety

Anxiety

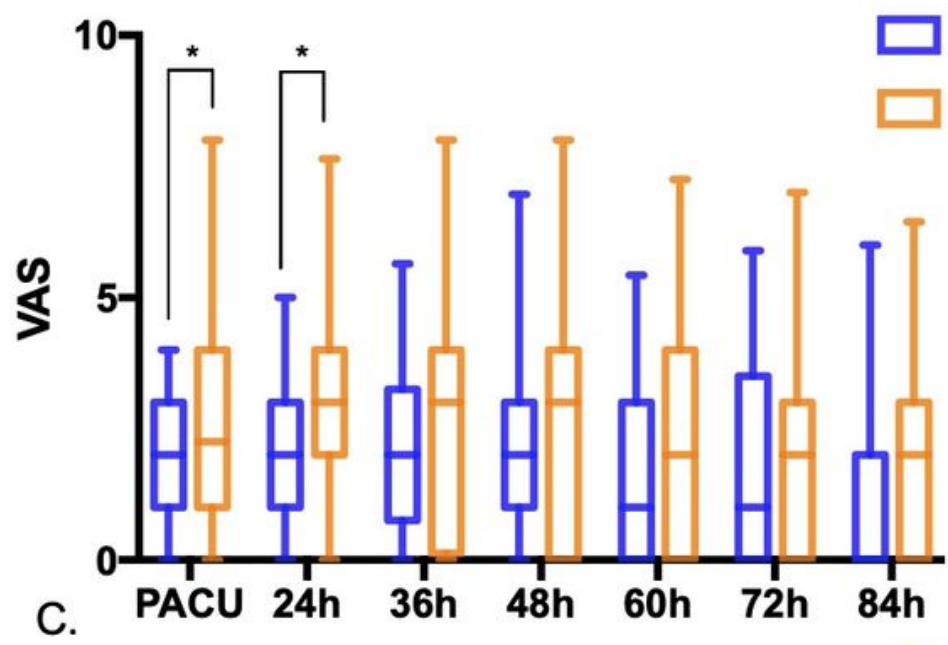

Non-depression

Deprssion

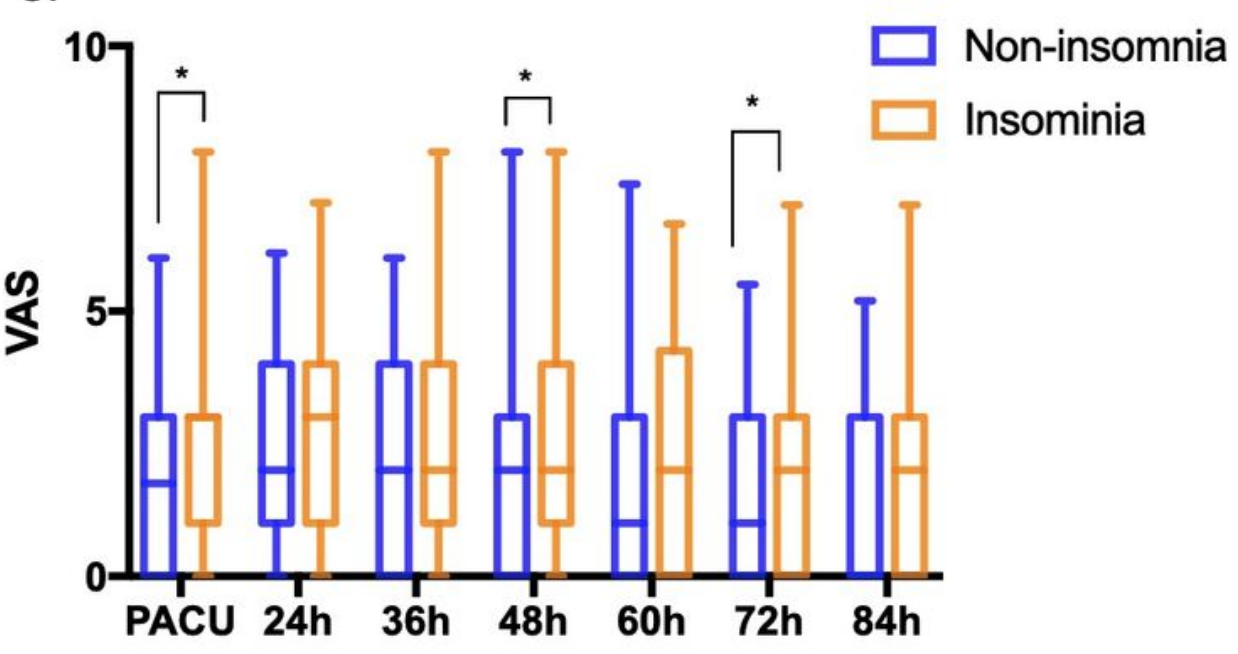

Figure 2

Comparison of postoperative VAS of patients after PSM. A. Comparison of postoperative pain between patients with and without anxiety after PSM. B. Comparison of postoperative pain between patients with and without depression after PSM. C. Comparison of postoperative pain between patients with and without insomnia after PSM. Data were expressed as median with interquartile range, and non-parametric 
tests were used. ${ }^{*} \mathrm{P}<0.05$ compared with patients without anxiety, depression or insomnia. VAS, visual analog scale; PSM: propensity score matching; PACU, postoperative anesthesia recovery room.

\section{Supplementary Files}

This is a list of supplementary files associated with this preprint. Click to download.

- plainengelishsummary.docx 